\title{
Dutch Moroccan Girls Performing their Selves in Instant Messaging Spaces
}

\author{
Koen Leurs and Sandra Ponzanesi
}

At around $10 \mathrm{pm}$ on a Tuesday evening in late January 2010, two 13-year-old Dutch Moroccan girls have a personal conversation. One would initially expect that such talk would be taking place over the phone. The girls can easily be pictured sitting in their bedrooms gossiping about a mutual friend. They appear to ridicule a girl they both know. However, after having a closer look at the transcript this idea must be thrown out of the window. Why do the speakers have such unusual names and what do the smileys (emoticons) hint at? And what do the girls allude to when they ask each other 'do you have her on msn'?

The medium that the girls are using is MSN Messenger, an instant messaging (IM) Internet application. MSN is a social media technology that allows individuals to privately exchange short messages and socialize, in real time. ${ }^{1}$

The conversation moves from an opening greeting sequence to Midia ('Triumph sweetie') asking whether 'miss mocro' is on speaking terms with another girl, Aisha. ${ }^{2}$ After finding out they are still quarrelling, Midia adds oil to the fire by gossiping about Aisha's 'display picture.' Aisha is a girl the correspondents both know, both from outside the world of IM ('I saw her today') and from within ('did you see that picture'). In the picture another girl, Yasmina, is apparently kissing Aisha. While Aisha 'looks 3ed [3ed: still, however] good' in the picture, the appearance of Yasmina irritates both girls. Miss mocro calls her names ('that whore') and types 'i hate yasmina.' She puts extra emphasis on her assertion by adding the term 'wollah,' meaning 'with Allah.' Midia, using capitalized letters, 'shouts' that she saw Yasmina on that day. In the end of their conversation, making use of smileys, the girls jokingly talk about whom they would want to have shot if they were given the option. The full conversation follows:

Triumph sweetie says: Hiiiwaaaaaaa

miss mocro says: Eeeeeeeeeeeeeeywaaaaaaaaa [ewa: now, yo] 
Triumph sweetie says: Whatsuuuuuuuup

miss mocro says: GoooooodGooooooooodwithyou?

Triumph sweetie says: Allriiighttttttttt

$: \mathrm{P}$

Haha

miss mocro says: $\mathrm{nXiiiice}$

Triumph sweetie says: ewaaaa howslife etccc

miss mocro says: badd

yours

Triumph sweetie says: Worse than yours i geus

miss mocro says: nooo cant bee

Triumph sweetie says: ohyess

wifeey

miss mocro says: nopp

Triumph sweetie says: ahha

ohwelll

are things still alright between you and aisha or noot

miss mocro says: nopp

Triumph sweetie says: pity

do you have her on msn

miss mocro says: yes

Triumph sweetie says: haha did you see that picture her and yasminaaaaaa

miss mocro says: that kiss?

Triumph sweetie says: wuhaha jhaa

miss mocro says: tfoee man that whore [tfoe: ugh, dirty]

Triumph sweetie says: aisha looks 3ed good but [3ed: still, however]

yasmina oh man

miss mocro says: $\mathrm{i}$ hate yasmina fo sure

wollah noot normal 8 [wollah: with Allah, I swear]

Triumph sweetie says: ISAW HER TODAY

Haha

Haha when they ask who would you like to shoot 
Triumph sweetie says: first yasmine than fayzal $\mathscr{B}$

miss mocro says: Ahhahaha wollahilaa

Triumph sweetie says: 8

miss mocro says: iwajaaa

This chapter examines how Dutch Moroccan girls construct their online selves using IM, focusing on gender as the primary analytical category with reference to other dimensions such as ethnicity, diaspora, youth culture, and religion. While particular digital communication systems come and go, IM has been around since the 1990s. ${ }^{3}$ Exploration of how youth engage with one another through IM provides important insights into the performance of selves and identity construction. IM can be understood as a private space where individuals in specific material-embodied contexts perform everyday, but very meaningful, symbolic-discursive work. We aim to make an empirical contribution to the performance of self through social media technologies while also refining theories of our understandings of the specificities of the IM medium, processes of adoption by its users, and IM digital-material expressive culture. We focus on two themes that arose from our corpus. First, the expression of hybrid belongings - which includes the perpetuation of cultural ideals of stereotypical masculinity and femininity - will be addressed. Second, IM will be shown to be a private space where migrant girls become gatekeepers and adapt the medium to become assertive cultural producers. However, we will first map out the literature on adolescent IM connectivity, situate our work theoretically, describe our methodological approach, and introduce our interviewees.

\section{Adolescent Instant Messaging Connectivity}

Instant messaging, available online since the 1990s, remains an important communication tool in the lives of many adolescent youth who are connected to the Internet. Lenhart, Rainie, and Lewis (2001) report that 'the majority of teenagers have embraced IM in a way that adults have not' (p. 10). In the context of the US, three out of four adolescents use IM frequently. IM is also especially used by girls as a space to socialize (Lenhart, Rainie, and Lewis, 2002, p. 38). Young people in the Netherlands consider MSN to be the most important communication technology for keeping in touch with others. In the Netherlands, 86 percent of all boys and 91 percent of all girls use MSN at least weekly and almost one in every two young people use it on a daily basis. Girls use MSN more than boys and use it for longer (Duimel and de Haan, 2007, p. 88). From our survey data, covering 346 Dutch Moroccan young people, we learned that IM technologies are also very popular in this group. Most girls (97 percent) and boys (93 percent) use the technology at least once per week, while 53 percent of girls and 43 percent of boys report logging in more than once daily (see Table 26.1). ${ }^{4}$ 
Table 26.1 Frequency of instant messaging usage (percentage): 'How often do you use Instant Messenger (MSN)?'

\begin{tabular}{lcccccc}
\hline $\begin{array}{l}\text { Frequency of } \\
\text { IM Usage }\end{array}$ & $\begin{array}{l}\text { I Day per } \\
\text { Neek or Less }\end{array}$ & $\begin{array}{l}\text { 2 or 3 Days } \\
\text { per Week }\end{array}$ & $\begin{array}{l}\text { 4 or 5 Days } \\
\text { per Week }\end{array}$ & $\begin{array}{l}\text { Once } \\
\text { per Day }\end{array}$ & $\begin{array}{l}\text { More than Once } \\
\text { Every Day }\end{array}$ \\
\hline Girls & 3.3 & 5.6 & 9.4 & 10.6 & 18.3 & 52.8 \\
Boys & 7.2 & 3.6 & 9.0 & 13.9 & 23.5 & 42.8 \\
\hline
\end{tabular}

The question arises as to why this technology seems especially to appeal to young people. Albero-Andrés (2004) asserts that IM use is a regular evening activity among adolescents. She adds that IM has 'replaced the long telephone conversations between friends that used to be so frequent in adolescence' (p. 112). As such, the communication now takes place in a new vehicle, but the topics that are important during this period of life have not changed. Similarly, Boyd (2010) argues that online spaces, in comparison with shopping malls and school yards, can be seen as gathering spots where youth like to hang out. She adds, 'while the site teens go to gather at has changed over time, many of the core practices have stayed the same' (p. 80). Unlike at physical gathering spots, adult supervision is often not found in IM spaces.

Bradley (2005) links the importance of personal autonomy during adolescence to the Internet. She states: 'adolescence is marked by the desire for autonomy and independence' and recognizes that 'the Internet offers adolescents social, moral, recreational, and intellectual experiences that are not mediated by adults' (p. 62). Grinter and Palen (2002) found that IM enables young people to remain connected away from adult supervision while being confined to their homes: 'IM is used to talk with friends outside the times that would be allowed either by natural constraints or by socially-determined constraints' (p. 26). In domestic settings, IM can be operated 'below the radar' and 'use can be unobtrusive, go unnoticed, or even be covert' (p. 26). This observation may help us to understand why in one study on youth in the US over one third of participants reported to have said something over IM that they would not have said elsewhere (Lenhart, Rainie, and Lewis, 2001, p. 22).

Everyday interaction with peers becomes mediated through the use of IM. Boneva et al. (2006) argue that the popularity of IM can be explained by two 'types of adolescent peer connectedness,' as it is compatible with one-to-one and one-tomany communication. An example of the first type is that youth can engage in synchronous one-to-one conversations with peers. During these conversations, the 'display picture' feature enables youth to upload an avatar or actual photo, which can be used to test out and discuss appearances. One-to-many communication takes place, for instance, when users broadcast their interests through the 'display name' feature, which is rendered visible to everyone on their 'buddy list.' Boneva et al. elaborate on the two modes of connectedness, describing them as the private disentangling of the self and the more public negotiation of group belonging as performed in IM: 'through maintaining individual friendships (that help them 
"decipher" the self) and through belonging to peer groups (that help them map the self onto the social categories of the larger world)' (p. 202). In a related example, Smale and Greenberg in their study of 444 users' IM display names found three themes that illustrate types of adolescent connectedness: 'identification ("who am I?"), information about self ("this is what is going on with me") and broadcast message ("I am directing information to the community")' (2005, p. 95). These themes illustrate how IM features are taken up both as personal and collective identity markers.

\section{The Co-construction of Instant Messaging}

Three general strands can be identified in the literature on embodiment and the Internet. There are two extremes - the utopian and the dystopian perspectives and an in-between perspective (van Doorn and van Zoonen, 2009). The utopian approach conceptualizes gender in terms of online identity construction. Cyberspace is seen as a liberating world disconnected from the offline world. Gender and also ethnicity/race, age, and looks are potentially absent or alternatively fabricated (Reid, 1993; Turkle, 1997). The dystopian approach dismisses information and communication technologies as standardizing exploitative neoliberal, default white, masculine, and heteronormative norms (Nakamura, 2002; Herring, 2003). However, the actuality lies somewhere between these extremes. A third strand therefore disapproves of both extremes and accepts that our ideas about gender, ethnicity, diaspora, and identity continue to be constituted and reconstituted every time we log on. The Internet is thus seen as part of everyday real life, where offline and online spheres infuse each other with meaning. IM performances are co-constructed at the crossroads of bodies, technologies, and discourses. When we articulate ourselves, the self is thus partly inscribed by the medium, partly interpellated in discursive norms, and partly shaped by the users' action (Wajcman, 2010).

The use of the Latin alphabet in IM to write Arabic brings our attention to how IM practices are influenced by the medium but also shaped by other dimensions such as the purposeful appropriation of technology by users for expressing a sense of identity. In the introduction we saw how Midia said Aisha looked '3ed good,' or 'still good,' in the picture, and later Midia emphasized a statement by typing 'wollah,' or 'with Allah.' These are two examples of the use of the Latin alphabet to write Arabic. Palfreyman and Al Khalil (2003) analyzed the representation of Arabic in IM conversations in the United Arab Emirates. Similarly to our case, their interviewees, female university students, combined characters from the Arabic alphabet with characters from the Latin alphabet to write Arabic in their IM communication. They found that employment of the Latin alphabet instead of the Arabic alphabet is shaped by 'linguistic, technological and social factors.' Using Latin characters is partly attributed to the influence of the American Standard Code for Information Interchange (ASCII) on online communication, which 
Palfreyman and $\mathrm{Al}$ Khalil recognize as 'a kind of lingua franca of the Internet' (2003). There is a general lack of support for Arabic script in keyboards, computers, and operating systems. Globally, the ASCII computer character set is the technological default. The standard mainly covers Latin letters, which are most commonly used in European languages, and excludes Arabic script (among other non-Latin scripts).

However, the practice of using Latinized Arabic, dubbed 'ASCII-ized Arabic' by Palfreyman and Al Khalil (2003), is not only shaped by constraints of computer character sets. ASCII-ized Arabic has also been taken up as an everyday informal writing style. In their casual IM conversations with peers, the United Arab Emirates female university students participating in the study by Palfreyman and Al Khalil wrote in ASCII-ized Arabic because of its 'ease of typing.' However, they also reported that they used it because of privacy concerns (their parents would not be able to follow the conversation) and because they were interested in 'writing in an unusual script.' Typing in AA, they negotiate between localized, linguistically specific vernaculars and Modern Standard Arabic. Linguistically specific vernaculars in the United Arab Emirates were previously only used for genres such as poetry and cartoons. They are now actively transferred and translated for the purpose of everyday informal computer-mediated communication. This writing style has grown into an informal and generationally specific symbolic resource invested with social meanings. The style is used to articulate a bounded collective identity with an 'in-group' that recognizes its principles, and allows its users to exclude 'outsiders' such as teachers and other adults.

\section{Identity Performativity in Instant Messaging}

Judith Butler famously deconstructed the category of gender by foregrounding the fact that gender is to be understood as something we do rather than something we are. There is no preceding or following ' $I$ ' that exists apart from gender performativity; rather, the 'I' comes into existence through a matrix of power relations. With her notion of performativity, Butler goes beyond distinctions between materialembodied and symbolic-discursive domains. Gender performativity is the constitutive stylized repetitious process through which one acquires subjectivity: 'language sustains the body not by bringing it into being or feeding it in a literal way; rather, it is by being interpellated within the terms of language that a certain social existence of the body first becomes possible' (Butler, 1997, p. 6). In order to render ourselves intelligible, gender, ethnic, age, and diaspora axes of identification have to be constituted through the citation of norms. However, in the articulation of identities, people are bounded by but not fully determined by such norms.

As we have seen in the discussion between Midia and 'miss mocro,' IM users re-mediate themselves through text. Identities are typed with special symbols, pauses, and smileys. Interviewee Inzaf compared her experience of IM to other means of communicating with peers: 'Via msn you don't know how the other is 
feeling and on the phone or when meeting in real life you do know that.' Speakers have to do without physical cues, and smileys, symbols, capitalization, abbreviations, Latinized Arabic, and netspeak answer the need for contextualization cues. In the words of another interviewee, Naoul, smileys are helpful in 'show[ing] the emotion that you have at that moment' (for example). Fatiha, a further participant, affirmed their specific value: 'in this way you show how you react on something it is easy to do this with an icon more easy than typing.' As our interviewees indicate and as we saw with the example of ASCII-ized Arabic, restriction to computer character sets and the creative appropriation of characters do not prevent users from making sense of one another. On the contrary, the absence of facial expressions and gestures has resulted in the emergence of a distinctive, creative expressive culture.

In the context of this study, the following three theoretical assumptions are key. First, the multiply located identities of our interviewees are not neutrally, naturally occurring entities but are actively constructed and made, in a process of both external ascription and internal claiming. Second, the interviewees' identity performativity results from encounters between individual bodies, technologies, and discursive frameworks. Third, the process occurs through the exchange of digital material artifacts such as display pictures, buddy lists, display names, and conversations.

\section{Entering a Private Space}

'Via msn you often dare to say more than when you have someone on the phone or [in English] face to face,' stated 17-year-old Dutch interviewee Fatiha. In the Netherlands, girls with a Dutch Moroccan background have been recognized as avid Internet users. They have turned to online spaces to challenge traditional, passive images of Muslim women. 'Dutch-Moroccan girls are more restricted in their freedom of movement than boys, and thus, the Internet widens their horizons' (Brouwer, 2006). These girls sometimes lack access to informal meeting places, as they have to negotiate between opposite motivational forces of continuity and change. According to Durham (2001a), 'adolescence, as experienced by girls of immigrant diaspora groups, is complicated by issues of race, culture and nation that intersect with discourses of sex and gender' (p. 140). Therefore, we will filter our observations using the lens of intersectionality. Although personal experiences among our participants varied, Dutch Moroccan boys are often 'allowed a wider radius of action outside the house' while 'girls still face the most restrictions, and they spend much of their leisure time with female family members and friends' in domestic settings (Pels and de Haan, 2003, p. 61).

This study details how Dutch Moroccan youth are active users of the private space of IM. There, they type themselves into being in distinct ways. Everyday discussions of personal issues, emotional support, fights, gossip, and flirting take place. Such activities generate fascinating insights into the performance of self in a 
digital space. However, entrance to this private space is not straightforward (Jacobs, 2005; Thiel-Stern, 2007). We believe this is why in-depth studies examining the expressive culture of IM are scarce, while research on publicly accessible digital spaces, for example social network sites such as Facebook and microblogs such as Twitter, are very common. Especially outside the US, studies on IM are few and far between.

\section{The Study}

The six young people - Fatiha, Inzaf, Kamal, Khadija, Naoul, and Midia - who took centre stage in this study were contacted through a snowball sampling method. Most became involved in our project after being introduced to us by a student who knew them personally. A Utrecht University student of Dutch Moroccan descent invited youth in her social circle to participate. In other cases, participants were invited on a personal level through a volunteer job in the community. We did not aim for a representative sample. The snowballing approach has its pros and cons. We depended, for instance, on a student as a key informant to gain access to the social worlds of our interviewees, which generated a set of participants living in two large cities in the Netherlands. Additionally, the snowball approach resulted in a sample of five girls and one boy. For our analysis, we chose to include all their voices in this chapter, as each participant allowed us a glimpse into their personal IM networks by sharing conversations with five of their contacts; their IM networks included youth of different gender and ethnic backgrounds.

Inspired by the pioneering work of Jacobs (2005) and Thiel-Stern (2007) on IM use among US adolescent girls, we adjusted our methods to meet the everyday digital media use of our six interviewees. This resulted in a mixedmethod set-up, including interview sessions through IM and via email, and occasional face-to-face meetings (Turkle, 1997). Additionally, the interviewees completed an online questionnaire, ${ }^{5}$ and in some cases a researcher was present to answer possible questions although in most cases the teenagers answered the questionnaire on their own. Interviews and IM transcripts were gathered over the period December 2009 to February 2010. Interested youth were asked to save IM transcripts of conversations with five people of their choice. From these logs youth were asked to select five conversations that they would like to share with us.

In our instructions, we emphasized that the topic of the conversations did not matter to us. To a certain extent it is likely that the teenagers sent in (parts of) conversations that would represent themselves in ways that seemed to them to be fitting for a university research project. In our research protocol, we set two conditions that had to be met for conversations to be submitted to us. First, the six participating young people were requested to ask their contacts' permission to share the transcript with us for our research at the beginning of their conversations. 
Second, the conversations had to comprise at least 10 turns. In practice, for example, Inzaf got permission from her contact as follows:

El Hoceima is the bom, that's the place where $i$ come from so just tell everyone thats the city number ONE!!! says: will you allow me to copy and paste this conversation for a msn research?

EEsmaa says: yea course sweety

As a small token of appreciation, our interviewees were given gift cards of 7.50 euros after they finished the questionnaire and the interviews. Following the data gathering phase, email and IM were used to ask questions to clarify the conversations and their contexts. The data-gathering efforts resulted in six interviews with the interviewees, six completed surveys, and 26 IM transcripts ranging in length from under a single printed page to over three pages. ${ }^{6}$ Not all interviewees succeeded in gathering the five requested logs in time. In sum, the corpus consists of over 70 pages of IM transcripts, interviews, emails, IM conversations, and survey data, which allow us an insider's glance at previously unheard personal narratives.

Reflexivity is important within feminist scholarship and we would like to acknowledge the influence of us as researchers on the data gathering and analysis. Koen Leurs did most of the data gathering, while Sandra Ponzanesi and Koen Leurs jointly analyzed the data. The gathering and analysis of the corpus were thus filtered through our perceptions and theoretical lenses. The situated knowledge that we have produced is located within the fields of feminist technoscience, postcolonial theory, and new media studies. We acknowledge our 'partial' perspectives and aim to be 'modest witnesses' (Haraway, 1997) to the expressive culture of IM. We aim to sketch a rich picture of our interviewees' everyday use of IM by triangulating different approaches and different sorts of data.

\section{Meet the Interviewees}

Our interviewees were aged between 13 and 18: Midia was 13, Inzaf and Naoul were 15, Kamal was 16, Fatiha was 17, and Khadija was 18. First and foremost we would like to emphasize that Fatiha, Inzaf, Kamal, Khadija, Naoul, and Midia were all from Dutch minority backgrounds and were urban youth. All had ties to Morocco and all their parents migrated to the Netherlands from Morocco from the 1960s onwards: they were part of the Dutch Moroccan community. With around 350,000 people, the Dutch Moroccan community is the second largest minority group in the Netherlands (2.1 percent of the population), after people with a Turkish Dutch background (Centraal Bureau voor de Statistiek, 2010). All our young participants spoke Dutch at home, in combination with either Arabic or a Berber language, and all participants considered themselves religious although Islam played varying roles in their lives. They all had access to the Internet in their homes; some had to share a computer with their sibling(s) while others had a computer in their bedrooms. 
We asked interviewees to save examples of IM conversations with their personal networks of friends. We received transcripts of talks with contacts aged between 13 and 22. Seven of the transcripts were conversations with boys and 19 were conversations with girls. These friends had a variety of different backgrounds. The participants used labels such as Dutch, Moroccan, Somalian, and Turkish to describe their friends. The diverse makeup of these IM friend networks illustrates a sense of online conviviality as conversations with people of various backgrounds are held in this single communicative space (Leurs, 2012). Next to ethnic labels, gendered and geographical markers of closeness were often used. Common markers used to describe contacts were 'she is a girl from my neighborhood,' 'he lives in [city],' and 'that is a boy from my class.' All but three transcripts were conversations with people who lived in the same city or a neighboring town. This indicates that the majority of contacts were also known in real life from everyday encounters such as school and work.

\section{Hybrid Belongings: Gender, Ethnicity, and Youth Culture}

Display names appear in the buddy lists of friends as part of an expressive culture full of one-to-many broatcasted emotions. Van Doorn, Wyatt, and van Zoonen (2008) state that nicknames are crucial in online identity performances: 'they can be used to display information that contributes to the performance of one's age, sex, location, and body type' (p. 364). When analyzing the transcripts, we saw that youth frequently used explicitly gendered display names and signed in with similarly gendered email addresses, for example including 'chick,' 'girl,' 'lady,' 'miss,' or 'boy' in Dutch, English, and other languages.

Users often went beyond gendered articulations of the self, however. In our corpus, display names revealed expressions of hybrid belongings, for example involving the expression of gender, ethnicity, and diaspora identifications. Interviewee Inzaf went by the display name of 'El Hoceima is the bom, that's the place where i come from so just tell everyone thats the city number ONE!!!' Thus, she showed her transnational affiliations, translating her belonging to the Moroccan diaspora into a rap rhyme. The display name 'miss mocro,' discussed in the introduction, combines a gendered term with a local ethnic affiliation. Mocro, an honorary nickname for Dutch Moroccan, is a term borrowed from Dutch Moroccan hip-hop culture, which entered the mainstream Dutch youth culture after Ali B's song Leipe Mocro Flavour reached the top of the charts in the Netherlands in 2005. A 15-year-old Dutch Moroccan boy used the name 'Mø مح م' BadBoy,' combining English words with his name in Arabic, which is also the name of the prophet. In the use of 'Mø' and 'miss mocro,' the symbolic value of the use of English lies in its connection to a global popular youth culture. The use of Arabic alphabet characters for the prophet Mohammed, or his own name, alongside 'Mø' provides a potential connection to the wider Islamic ummah ('community').

As mentioned, 'miss mocro' and 'Mø Р२?: BadBoy' reference mainstream global hip-hop youth culture. Kaya (2002) focused on migrant boys in Berlin and posited that hip-hop youth culture 'enables ethnic minority youths to use both their own 
"authentic" cultural capital and the global transcultural capital in constructing and articulating their identities' (p. 165). Discussing display names with our interviewees revealed further gendering of display names. Kamal observed that his male friends often took 'a piece of a rap or a song.' Fatiha used the name 'life does not always happen the way you want it,' and she explained that girls typically have cute and sweet things in their display names while boys include tough elements. Inzaf agreed and reported about boys that 'most that I know take a line from a well known rap song' and girls put down who they are in love with or include little poems. She, for instance, went by the display name of 'let the past rest and wake up the future.' These findings resonate with earlier studies on IM. For instance, in their study with Taiwanese participants, Chou and Chen (2009) observed gender differences in IM display names: 'all female participants tended to disclose sentimental nicknames with the goal of complaining about something' (p. 124).

Young people who use IM often maintain long lists of contacts; those of our interviewees range between 50 and 300. Midia had 300 contacts, Kamal had 164, Fatiha had 160, Naoul had 150, Inzaf had 70, and Khadija had 53. In articulating display names, 'digital labour' (Nakamura, 2009) is performed to grab the attention of peers and gain status. An effort is made to attract attention. The young people who participated in the study of Lewis and Fabos (2005) were constantly monitoring their buddy lists, as friends in these lists regularly changed their display names. Lewis and Fabos observed that the buddy list is used as one of the 'surveillance features' of the program (p. 489). Young people keep an eye on the various ways in which their friends author themselves. Our case study reveals that this naming is gendered but also displays hybrid forms of religious, ethnic, and youth cultural belonging.

From display names we now turn to the use of the 'display pictures' feature. When we asked young people in our survey how they would show themselves in their display pictures in order to be liked by their friends, the Dutch Moroccan respondents provided us with interesting insights into their perceptions of selfpresentation practices (see Table 26.2). ${ }^{7}$ Most importantly, we found that almost half of the girls ( 49 percent) and nearly half of the boys ( 43 percent) in our survey sample reported that they preferred to present themselves as 'normal.' Striving to meet expectations of what is accepted as normal and standard in IM expressive culture seems important. In addition to the label 'normal,' boys and girls chose specific labels to describe the self-presentation to which they aspired. Commonly chosen labels indicated that the girls who participated wanted to look 'sweet' (47 percent), 'trendy' (22 percent), or 'social' ( 14 percent), while boys wanted to look 'sporty' (36 percent), 'tough' (27 percent), or 'rich' (15 percent). Interestingly, being 'attractive/sexy' was listed more often by boys than girls ( 23 percent versus 12 percent). ${ }^{8}$ During our interviews, Inzaf suggested that girls show 'most of the time pictures of their lips or of themselves,' while boys show 'pictures of themselves with their friends and sometimes of their sixpack.' 17-year-old Fatiha adds that girls use 'mostly nice sensual or emotional images,' while boys use 'mostly tough looking pictures.' 
Table 26.2 Self-presentation labels: 'How would you show yourself in your picture in order to be liked by your friends?'

\begin{tabular}{lcc}
\hline Self-presentation Labels & Percentage of Girls & Percentage of Boys \\
\hline Intelligent & 11.1 & 12.7 \\
Sweet & 47.2 & 15.1 \\
Thoughtful & 9.4 & 26.5 \\
Rich & 3.9 & 15.1 \\
Normal & 48.9 & 43.4 \\
Computer savvy & 2.2 & 7.2 \\
Sporty & 8.3 & 36.1 \\
Beautiful & 46.7 & 23.5 \\
Attractive/sexy & 11.7 & 22.9 \\
Social & 14.4 & 9.0 \\
Rebel & 1.7 & 1.8 \\
Hard working & 1.7 & 7.8 \\
Trendy & 21.7 & 1.8 \\
Nerd & 0.6 & 2.4 \\
\hline
\end{tabular}

What we observe here is a particular localization of commercialized global youth discourses: a perpetuation of the dichotomy of clear-cut masculinity versus sensual femininity, and rough-and-toughness versus affectivity. Further, the fragmentation of the body into sexualized objects - seductive lips for girls and well-muscled abdomens for boys - reflects idealized stereotypical images of adolescent girls and boys. Preoccupations with physical beauty myths are typical for Western adolescent youth, as is their consumption of perfect sexual body discourses. The latter, for instance, find resonance in the literature on US adolescent girls' messenger use (Durham, 2001b; Thiel-Stern, 2007, p. 85). Thiel-Stern (2007) argues that US adolescent girls consume ideals of culturally defined female identities on IM: 'IM is a space as guided by corporate and commercial discourses as any magazine or television show' (p. 97). Throughout our research in the months of January and February 2010 we looked at the 'MSN today' pop-up window, which automatically appears when signing in. The pop-up window is a piece of virtual real estate used by corporations to attract IM users to their advertisements, with common topics such as dieting ('lose weight like Beyonce'), celebrity gossip ('Brad and Angelina break up,' 'Dinand frank about cheating'), and sex tips ('Trend: a sexy 2010,' 'Prima donna's in porn') that further play into stereotyping gender, sexuality, and body myths.

Corporations are seeking entry into the private space of IM. Pop-ups, IM bots (automated IM partners added to one's contact list that offer information about banking, shopping, etc.), and commercials appear within the personal space (ThielStern, 2007, p. 100). Young people in our study generally paid little attention to advertisements on MSN. Fatiha, for instance, told us: 'I ignore those commercials and they do not bother me.' Merskin (2005) calls girls that choose to express 
themselves and resist dominant discourses 'jammer girls.' Jammer girls, as cultural producers, make use of sociotechnological changes consistent with third-wave feminism to take control of their own expressive culture by using Internet features such as interactivity, self-publishing, and critical consumption (p. 56). ${ }^{9}$ Advertisements are tolerated as a necessary evil, without which the free IM service would probably not exist.

This is but one side of the story that needs to be told about IM. Similarly to how social networking sites such as Facebook blur boundaries between calculated corporate interests and fostering a youth-centered sphere, the interviewees showed that IM expressive culture is a contested space. Another side to the story of IM also demands our attention: the assertive potential of IM users is illustrated in their ability to dismiss advertisements within their personal spaces as well as act as gatekeepers to those personal spaces.

\section{'MSN is for Yourself': Gatekeeping Spaces of their Own}

This section explores how our interviewees appropriate IM as a private space. IM enables youth to privatize their communication, to keep it 'below the radar' (Grinter and Palen, 2002, p. 26). The IM transcript below is an example of talk about boys. 17-year-old Fatiha is in conversation with her 22-year-old Dutch Somalian classmate Owsark. Before turning to the sensitive topic of boys, Owsark makes sure they are talking privately, on a one-to-one basis. This gesture can be compared to the offline world, when it is sometimes necessary to make sure that nobody is overhearing your conversation before saying something.

owsark says: ohyea theres something are you alone

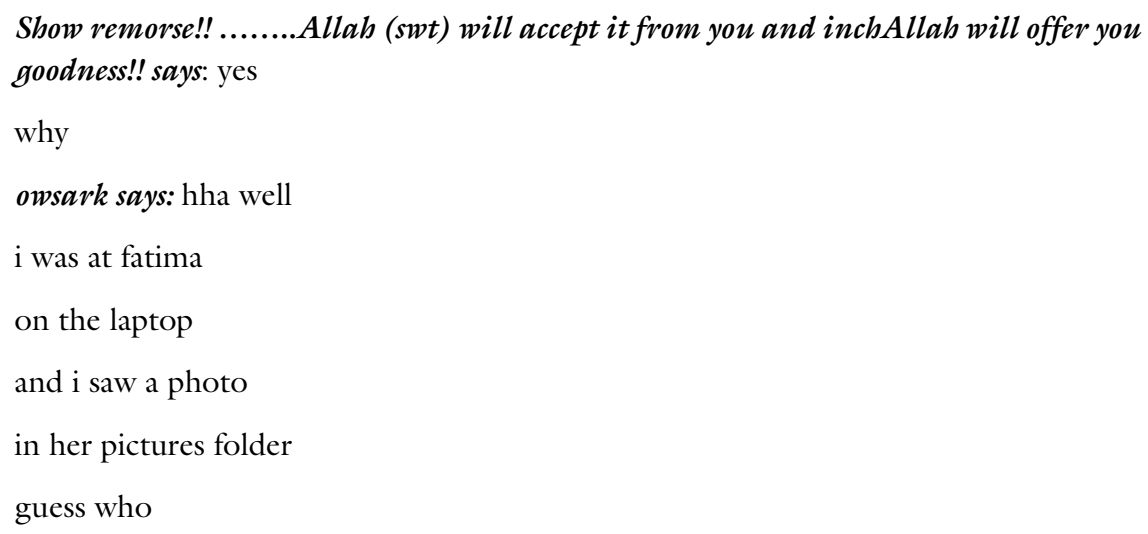

Show remorse!! ........Allah (swt) will accept it from you and inchAllab will offer you goodness!! says: who?

owsark says: that one guy i told you about

i told her what are these photos doing here 

Show remorse!! ........Allah (swt) will accept it from you and inchAllab will offer you
goodness!! says: which ones?

owsark says: she know him

the guy in school

our the one you interviewd

\begin{abstract}
Show remorse!! ........Allah (swt) will accept it from you and inchAllab will offer you goodness!! says: oooh khalid

hahahaha
\end{abstract}

owsark says: yes that one

wallah saw [wollah: with Allah, for extra emphasis]

a picture of him

Owsark explains that she has discovered photos of a boy she likes in the pictures folder on the computer of a mutual friend. It appears from the text that Owsark is somewhat jealous. She wants to know from Fatiha how she thinks the photographs got there. She quickly fires a series of short questions about the girl: 'she know him'? 'the guy in school'? 'the one you interviewd'? As observed in display names and in conversations, MSN is often used to talk with, and about, boys. Naoul states that girls put boys' names in their display names and 'when they have a boyfriend they show the date of their relationship.' Others include more implicit references such as 'Im Crazy in Love with you my feelings for you cant go away 8 '

In Midia's words, MSN 'is for your self, nobody sees who and how many contacts you have in your list.' Affiliations that are built within this space can be kept personal; they are only for the eyes of the individual user. Outsiders cannot see who is on another's contact list and who is not. Brouwer found that Dutch Moroccan girls turn to the Internet to overcome intersecting issues of gender, religion, and sexuality. They contact people 'without the social control of parents and without crossing social boundaries.' Brouwer witnessed that themes of love, Islam, marriage, and sexuality were especially heavily discussed (2006). What we see here is that these girls are sometimes seen as 'upholders of collective traditional values' (Ponzanesi, 2002, p. 210). The social confines of the community are not as stringent on the Internet: 'Personal contact is difficult in our culture; you never know who you can trust' (interviewee, cited in Brouwer, 2006). IM has been taken up as an alternative site for social peer bonding, as engaging in contact with the opposite sex in full view of parents and the community can sometimes be difficult for these girls.

Importantly, youth using IM exert a great degree of control over whom they want to engage with through the medium. However, Naoul thought that people are right in stating that MSN can be dangerous as total strangers can try to get in contact: 'it is possible that people you don't know add you to their contact list'; youth must thus remain active gatekeepers to their own space. Midia explains: 'you can have people stalking you, such as people adding you and telling you they know 
you or something like that, then you just have to block and delete.' These girls illustrate how they have authority over their turf; they get to decide who is included and who is excluded.

Herring found that online communication often discriminates against women. She suggested that moderators' control of interactional norms gives women greater influence as it results in more active participation (2003, p. 209). In IM, youth can moderate their own personal space through an intricate process of boundary making. Their attempts at securing greater autonomy in IM indicate that it has become a site for exploring and building intimate relationships: 'Since it is not appropriate for a Muslim girl to go to a café to meet the opposite sex, the Internet functions as a protected meeting place $[. .$.$] the advantage of going online is that a$ girl does not cross any sexual boundary' (Brouwer, 2006). Our interviewees agreed that the private space of IM allows for a relatively safe and non-compromising way to establish intimate relationships. In the words of Midia: 'I think that every girl first talks to a boy on MSN and get to know each other better and then make plans for a date.' Girls can safeguard their own IM spaces; when contacts become unwanted conversation partners, they can be removed from the list.

\section{Conclusions}

Since the 1990s, IM has been immensely popular among youth. IM goes back to the purely text-based roots of the Internet, but it has remained a relatively understudied and under-theorized social media technology because it is not straightforward to gather data within this private space. This study on IM expressive culture provides a window into the private and personal engagements of teens with their peers. As Brouwer argues, studying the Internet may 'uncover the invisible voice of marginal groups, particularly women' (2006). Six Dutch Moroccan youth have granted us access to their personal MSN communication network. With the help of Fatiha, Inzaf, Kamal, Khadija, Naoul, and Midia, we have witnessed how migrant girls perform themselves and construct their identities using IM. We analyzed a corpus of interviews, survey data, and IM transcripts to understand how youth write themselves into being in these spaces.

Building on Butler's theoretical toolkit on gender identity performativity, our focus has been on the intersectional constitution of selves through language in its wider context of power relations. By combining this perspective with an emphasis on technological performativity, we examined situated, embodied experiences of IM users. In IM, gender and technologies intersect with and influence each other. We wanted to take into consideration how the applications' interfaces, computer character sets, and commercial incentives inscribe themselves upon the users' performance of self. We also wanted to be aware of how users adapt to the environment. Therefore, we sketched a middle ground between mythic utopian and dismissive dystopian perspectives on digitized embodiment. This third perspective - acknowledging the 
co-construction of users, discourses, and technology - enabled us to remain sensitive to both digital structures and discursive norms.

We found that IM carries with it gendered social dynamics. Feminist media research insists that ideologies persisting in mass culture play a substantial role in the performance of gender. This study demonstrates that such broader social phenomena also inform the ways in which adolescents author themselves online. In conversations, display names, display pictures, and advertisements, cultural stereotypical femininities and masculinities are consumed and performed. However, youth also perform their individuality by diversifying their performance using youth-cultural, ethnic, religious, diaspora, and netspeak affiliations.

Moreover, by setting the boundaries of who is included and who is excluded in their buddy lists, by dismissing advertisements, and by becoming active agents of their own representations, our interviewees expressed how they are active gatekeepers of their own space. Further, our interviewees took up IM as a site for exploring and building intimate relationships by seizing the opportunity to safely engage with boys and girls without having to meet face to face. In sum, IM expressive culture is significant for our understanding of migrant girls online in the interaction with private/public spaces, the construction of selfhood, negotiating issues of friendship, and the production and consumption of sexuality.

\section{Acknowledgements}

We would like to express our gratitude to the interviewees, who openheartedly shared their IM experiences with us. Also, we would like to thank Fayrouz Boulayounne and users of the Maroc.nl online discussion board community, who provided us with additional translations and clarifications of Arabic, Islamic, and Berber words and statements.

\section{Notes}

1 In 2005, MSN Messenger was renamed 'Windows Live Messenger,' but here we use the name commonly used by our respondents.

2 While interviewees' names have been changed, original display names are included; they cannot be traced to individual users.

3 Consider, for example the decline of MySpace, once synonymous with online social networking but in the mid-2000s overtaken in popularity by the now more fashionable Facebook.

4 For our larger Utrecht University research project, 'Wired Up,' in which we assess digital media as innovative socialization practices for migrant youth, a computer-based, online questionnaire was conducted. The survey was carried out over the period fall 2009 to summer 2010, in various secondary schools in urban areas of the Netherlands, among 1353 participants. The interviewees who are introduced in this chapter participated in the piloting of the survey. Table 26.1 and Table 26.2 were generated from the answers 
of 346 young people of Dutch Moroccan descent who completed the survey. They were aged between 10 and 20 and were from various educational backgrounds. The sample was equally distributed, with 52 percent girls and 48 percent boys.

5 See note 4.

6 A note on translation: before the analysis was carried out, the gathered data were translated into English. In computer-mediated communication, spelling, grammar, and norms are considerably different from the rules youth are expected to obey when writing in school contexts. In an attempt to reduce intervention as well as to convey to the readers some of the original multi-lingual dynamics of the corpus, the often-included non-Dutch words and sentences (Spanish, English, Tamazight) were not translated into English. Translations and clarifications of these words are provided in the transcripts as bracketed text, while ' $[. .$.$] ' indicates that lines in the transcript have been left out. By$ asking participating youth to reflect on a selection of expressions and phrases, the rich character of the gathered data was further drawn out. To further reduce intervention, we sought ways to keep intact misspellings and netspeak jargon. These were carried over into English. For example, when in the transcripts or display names the Dutch word for 'girls' was spelled as 'meiisjes' (with an extra i), this was translated into English as 'giirls.' In this way we tried to capture at least some of the particularities of computer-mediated communication language play and migrant IM girl culture.

7 We allowed respondents to give multiple answers to this question, as we envisioned that display pictures would not carry single meanings for young people. This opportunity was used, as the percentages add up to well over 100 percent for both girls and boys. See note 4 .

8 The moral panic surrounding posing provocatively in photos circulating on the Internet is often superimposed on either 'bad' or 'innocent' girls.

9 As Snyder argues, embracing ambiguity, third-wave feminism is multiperspectival, remains attentive to multivocality, and deconstructs intersections of power relations in personal narratives $(2008$, p. 175). From this perspective on everyday, messy micropolitics, as recognized by Genz (2006), 'active consumption' within the capitalist economy can foster contradicting opportunities for agency and empowerment (p. 345).

\section{References}

Albero-Andrés, M. (2004). The internet and adolescents. In J. Goldstein, D. Buckingham, and G. Brougere (Eds.), Toys, Games and Media (pp. 109-129). Mahwah, NJ: Lawrence Erlbaum.

Boneva, B. S., Quinn, A., Kraut, R. E., Kiesler, S., and Shklovski, I. (2006). Teenage communication in the instant messaging era. In R. Kraut, M. Brynin, and S. Kiesler (Eds.), Computers, Phones, and the Internet: Domesticating Information Technology (pp. 201-217). Oxford: Oxford University Press.

Boyd, D. (2010). Friendship. In M. Ito (Ed.), Hanging Out, Messing Around and Geeking Out (pp. 79-115). Cambridge: MIT Press.

Bradley, K. (2005). Internet lives: Social context and moral domain in adolescent development. New Directions for Youth Development, 108, 57-76.

Brouwer, L. (2006). Giving voice to Dutch Moroccan girls on the internet. Global Media Journal, 5(9). Retrieved from http://lass.calumet.purdue.edu/cca/gmj/fa06/ gmj_fa06_brouwer.htm 
Butler, J. (1997). Excitable Speech. New York: Routledge.

Centraal Bureau voor de Statistiek. (2010). Population, core figures. Statistics Netherlands. Retrieved from http://statline.cbs.nl/

Chou, P. N. and Chen, W. F. (2009). Name-display feature for self-disclosure in an instant messenger program: A qualitative study in Taiwan. Issues in Informing Science and Information Technology, 6, 113-126.

Duimel, M. and de Haan, J. (2007). Nieuwe links in het gezin [New links in the family]. The Hague: Sociaal en Cultureel Planbureau.

Durham, M. G. (2001a). Constructing the 'new ethnicities': Media, sexuality, and diaspora identity in the lives of South Asian immigrant girls. Critical Studies in Media Communication, 21(2), 140-161.

Durham, M. G. (2001b). Adolescents, the internet and the politics of gender. Race, Gender \&. Class, 8(4), 20-41.

Genz, S. (2006). Third way/ve. Feminist Theory, 7(3), 333-353.

Grinter, R. E. and Palen, L. (2002). Instant messaging in teen life. In Proceedings of the 2002 ACM Conference on Computer Supported Cooperative Work (pp. 21-30). Retrieved from http://doi.acm.org/10.1145/587078.587082

Haraway, D. (1997). Modest_witness@secondmillennium. New York: Routledge.

Herring, S. C. (2003). Gender and power in on-line communication. In J. Holmes and M. Meyerhoff (Eds.), Handbook of Language and Gender (pp. 202-228). Oxford: Blackwell.

Jacobs, G. E. (2005). 'Ur Part of it': Portfolio People and Adolescent Use of Instant Messaging. Doctoral dissertation. University of Rochester, Rochester, NY.

Kaya, A. (2002). Aesthetics of diaspora: Contemporary minstrels in Turkish Berlin. Journal of Ethnic and Migration Studies, 28(1), 43-62.

Lenhart, A., Rainie, L., and Lewis, O. (2001). Teenage life online. Retrieved from http:// www.pewinternet.org/Reports/2001/Teenage-Life-Online.aspx

Leurs, K. (2012). Identity, Migration and Global Digital Media. Doctoral thesis. Utrecht, The Netherlands: Utrecht University.

Lewis, C. and Fabos, B. (2005). Instant messaging, literacies and social identities. Reading Research Quarterly, 40(4), 470-501.

Merskin, D. (2005). Making an about-face. Jammer girls and the world wide web. In S. R. Mazzarella (Ed.), Girl Wide Web. New York: Peter Lang.

Nakamura, L. (2009). Neda Soltani, race, and digital labor. In Difference Engines. Retrieved September, 28, 2010, from http://www.differenceengines.com/?p=189

Nakamura, L. (2002). Cybertypes. Race, Ethnicity and Identity on the Internet. New York: Routledge.

Ponzanesi, S. (2002). Diasporic subjects and migration. In G. Griffin and R. Braidotti (Eds.), Thinking Differently. A Reader in European Women's Studies. London: Zed Books.

Pels, T. and de Haan, M. (2003). Continuity and Change in Moroccan Socialization. Utrecht, the Netherlands: Verwey Jonker and Utrecht University.

Palfreyman, D. and Al Khalil, M. (2003). 'A funky language for teenzz to use': Representing Gulf Arabic in instant messaging. Journal of Computer Mediated Communication, $9(1)$. Retrieved September, 20, 2010 from http://jcmc.indiana.edu/vol9/issuel/palfreyman.html

Reid, E. (1993). Electronic chat: Social issues on internet relay chat. Media International Australia, 67, 62-70. 
Smale, S. and Greenberg, S. (2005). Broadcasting information via display names in instant messaging. In M. Pendergast, K. Schmidt, G. Mark, and M. Ackerman (Eds.), Proceedings of the ACM Group 2005 (pp. 89-98). Retrieved from http://portal.acm. org/citation.cfm?doid=1099203.1099218

Snyder, C. R. (2008). What is third-wave feminism? A new directions essay. Signs: Journal of Women in Culture and Society, 34(1), 175-196.

Thiel-Stern, S. (2007). Instant Identity. Adolescent Girls and the World of Instant Messaging. New York: Peter Lang.

Turkle, S. (1997). Life on the Screen. London: Weidenfeld and Nicolson.

van Doorn, N. and van Zoonen, L. (2009). Theorizing gender and the internet. Past, present, and future. In A. Chadwick and P. N. Howard (Eds.), Routledge Handbook of Internet Politics. New York: Routledge.

van Doorn, N., Wyatt, S., and van Zoonen, L. (2008). A body of text. Feminist Media Studies, 8(4), 357-374.

Wajcman, J. (2010). Feminist theories of technology. Cambridge Journal of Economics, $34(1), 143-152$. 